\title{
Equilibrium Space Charge Distribution in a Quadrupole Ion Trap
}

\author{
Shenheng Guan* and Alan G. Marshall* \\ Departments of Chemistry and Biochemistry, The Ohio State University, Columbus, Ohio, USA
}

\begin{abstract}
A simple model provides a basis for evaluating the ion spatial distribution in a quadrupole (Paul) ion trap and its effect on the total potential (trap potential plus space charge) acting on ions in the trap. By combining the pseudopotential approximation introduced by Dehmelt 25 years ago with the assumption of thermal equilibrium (leading to a Boltzmann ion energy distribution), the resulting ion spatial distribution (for ions of a single mass-to-charge ratio) depends only on total number of ions, trap pseudopotential, and temperature. (The equilibrium assumption is justified by the high helium bath gas pressure at which analytical quadrupole ion traps are typically operated.) The electric potential generated by the ion space charge may be generated from Poisson's equation subject to a Boltzmann ion energy distribution. However, because the ion distribution depends in turn on the total potential, solving for the potential and the ion distribution is no longer a simple boundary condition differential equation problem; an iterative procedure is required to obtain a self-consistent result. For the particularly convenient operating condition, $a_{z}=q_{z}^{2} / 4$ [in which $a_{z}=$ $-8 q U / m \rho_{0}^{2} \Omega^{2}$, and $q_{z}=-4 q V / m \rho_{0}^{2} \Omega^{2}$, where $U$ and $V$ are direct current and radiofrequency (frequency, $\Omega$ ) voltages applied to the trap, $m / q$ is ion mass-to-charge ratio, and $\rho_{0}$ is the radius of the ring electrode at the $z-0$ midplanel, both the pseudopotential and the ion distribution become spherically symmetric. The resulting one-dimensional problem may be solved by a simple optimization procedure. The present model accounts qualitatively for the dependence of total potential and ion distribution on number of ions (higher ion density or lower temperature flattens the total potential and widens the spatial distribution of ions) and pseudopotential (higher pseudopotential increases ion density near the center of the trap without widening the ion spatial distribution). ( 7 Am Soc Mass Spectrom 1994, 5, 64-71)
\end{abstract}

$\mathrm{P}$ articularly over the past couple of years, quadrupole ion trap (QIT, also known as Paul or Quistor ion trap) mass spectrometry has developed into a versatile and powerful techrique for mass analysis [1-3]. Demonstrated capabilities include wide mass range, multistage $\mathrm{MS}^{\mathbf{n}}$, and under special conditions high mass resolving power. However, mass measurement accuracy is much more difficult to achieve. Space charge effects constitute one of the most important factors contributing to mass measurement accuracy. Unfortunately, space charge effects are difficult to model theoretically and to control experimentally. Development of an effective mass calibration law that incorporates the effect of space charge is perhaps the greatest current challenge for analytical QIT mass spectrometry. We propose a simple model, based on combining Dehmelt's [4] pseudopotential approximation with a thermal equilibrium assumption for ion energy distribution. Our model offers a first step to-

Address reprint requests to Professor Alan G. Marshall, National High Magnetic Field Laboratory, Florida State University, 1800 East Paul Dirac Drive, Tallahassec, FL 32306-4005.

${ }^{*}$ Current address: National High Magnetic Field Laboratory, Florida State University, 1800 East Paul Dirac Drive, Tallahassee, FL 323064005 . ward future efforts to understand the effect of space charge effect on mass calibration in the QIT.

In a QTT, the sum of a direct current (DC) voltage and a high-frequency, high-amplitude radiofrequency (RF) voltage applied between the ring and endcap electrodes forms a three-dimensional time-varying electric potential that may be approximated as a quadrupolar electrostatic potential [4] if the corresponding $q_{z}$ value is small $\left(q_{z}<0.4\right.$; see Theory). In that limit, ion axial or radial motion may be modeled as a harmonic oscillator whose force constant depends on ion mass-to-charge ratio. Unlike a three-dimensional electrostatic potential in free space surrounded by closed-surface electrodes, the three-dimensional pseudopotential has a "true" energy minimum at the center of the trap.

Use of helium as a buffer gas has been shown to increase the mass resolving power and sensitivity of the QIT [5]. At a millitorr pressure of helium, ions collide with room-temperature He atoms several tens of thousands of times per second. Ion motions are therefore thermalized by the large numbers of collisions with room-temperature helium atoms. After an ion storage period of a few seconds, one can expect that ions will approach an equilibrium distribution [6]. 
Under typical working conditions in an actual QIT, the equilibrium assumption is certainly an imperfect approximation. However, when ions are located near the center of trap and the $q_{z}$ value is small, the motions of ions are smoothed by collisions with $\mathrm{He}$ gas atoms and we may treat a given ion as if spatial distribution of all the other ions is static.

\section{Prior Treatments}

Todd et al. [7] have evaluated prior theoretical models for the effect of space charge on QIT performance. Those models assumed a spatially uniform ion distribution (i.e., quadrupolar space charge potential) that is equivalent to an additional DC voltage on the electrodes $[8,9]$ ). Subsequently, Todd et al. [10] analyzed the ion spatial distribution in a QIT in the absence of space charge, by use of a phase-space dynamics approach. Vedel et al. [6] analyzed the ion threedimensional spatial distribution in a QIT in the presence of ion-neutral collisions but in the absence of space charge by a stochastic model. Vedel and Andre [11] and Vedel [12] later analyzed the effect of the space charge potential from an assumed Gaussian spatial distribution (in the limit of low ion number) as a small perturbation to the pseudopotential. In addition, Helmer [13] used the same spherical pseudopotential condition as in this article (see Theory), but treated the ion cloud as a sphere of uniform charge density at zero temperature, whereas we solve for the nonuniform ion spatial distribution at any Boltzmann temperature. None of these prior models is appropriate to a QIT containing a large number of trapped ions.

\section{Present Approach}

Under the assumption of equilibrium, the charge spatial distribution depends only on three factors: number of charges, pseudopotential generated by the voltages applied to the trap electrodes, and temperature (common for both ions and neutrals). Obviously, the assumption of equilibrium neglects any effect of the nature of the collision gas (e.g., helium versus argon), which would require a more detailed model. At equilibrium, ions must exhibit a Boltzmann kinetic energy distribution. The distribution of ions is then uniquely determined by the tutal electrostatic potential (i.e., pseudopotential plus potential due to space charge). However, evaluation of the space charge potential requires that the space charge distribution be known. Therefore, evaluating the spatial distribution of ions in a QIT is not a conventional boundary condition problem! We propose the following simple optimization procedure. First, an arbitrary charge spatial distribution is specified, and the space charge potential is computed for that distribution. The space charge potential is added to the pseudopotential, and the charge distribution is then regenerated subject to the new total potential. This process is repeated for many cycles until the charge distributions or the total potentials from consecutive iterations agree with each other. We then systematically analyze the behavior of the ion distribution or potential as a function of the model parameters: ion number, temperature, and QIT pseudopotential.

\section{Theory}

\section{Pseudopotential Approximation}

In a QIT, axial and radial motions of an ion of mass, $m$, and charge, $q$, are described by the Mathieu equations [1]:

$$
\frac{d^{2} u}{d \xi^{2}}+\left(a_{u}-2 q_{u} \cos 2 \xi\right) u=0 \quad \text { (S.I. units) }
$$

in which

$$
\begin{gathered}
u=x, y, \text { or } z \\
a_{z}=-2 a_{x}=-2 a_{y}=-\frac{8 q u}{m \rho_{0}^{2} \Omega^{2}} \\
q_{z}=-2 q_{x}=-2 q_{y}=-\frac{4 q V}{m \rho_{0}^{2} \Omega^{2}} \\
\xi=\frac{\Omega t}{2}
\end{gathered}
$$

in which $m$ is the mass and $q$ is the charge of the ion, $U$ is the DC voltage, $V$ and $\Omega$ are the RF voltage amplitude and angular frequency (both $U$ and $V$ are applied between the endcaps and the ring electrode), and $\rho_{0}$ is the radius of the ring electrode at the $z=0$ midplane of the trap. Typically (but not necessarily [14]), $\rho_{0}^{2}=2 z_{0}^{2}$, in which $2 z_{0}$ is the separation along the $z$-axis between the two endcap electrodes. The Mathieu equation has been studied in detail by McLachlan [15]. Simple analytical expressions for the solutions of the equation are not available, and its behavior is understood largely on the basis of a "stability" diagram [1], showing the boundary values of dc and if voltages at which ion trajectories are stable within the trap. In the stable regions, ion trajectories may be expressed as a linear combination of sine and cosine elliptic series:

$$
\begin{aligned}
u(\xi)= & A \sum_{-\infty}^{\infty} C_{2 n} \cos \left(2 n+\beta_{u}\right) \xi \\
& -B \sum_{-\infty}^{\infty} C_{2 n} \sin \left(2 n+\beta_{u}\right) \xi
\end{aligned}
$$

in which $A$ and $B$ depend on the initial conditions and $C_{2 n}$ and $\beta_{u}$ are complicated functions of $a_{n}$ and $q_{u}$. For example, the relationship between $a_{u}, q_{u}$, and $\beta_{u}$ may be expressed as:

$$
\begin{aligned}
a_{u t}= & \beta_{u}^{2}+\frac{1}{2\left(\beta_{u}^{2}-1\right)} q_{u}^{2} \\
& +\frac{\left(5 \beta_{u}^{2}+7\right)}{32\left(\beta_{u}^{2}-1\right)^{3}\left(\beta_{u}^{2}-4\right)} q_{u}^{4}+O\left(q_{u}^{6}\right)
\end{aligned}
$$


in which $O\left(q_{u}^{6}\right)$ represents all the terms of sixth order or higher in $q_{u}$ [15]. For small $q_{u}, \beta_{u}$ may be approximated as:

$$
\beta_{u}=\left(a_{u}+\frac{q_{u}^{2}}{2}\right)^{1 / 2}
$$

For small $\beta_{u}$ values $\left(\beta_{u} \ll 1\right.$ ), the ion trajectory, eq 2 , yields ion motions at a harmonic series of "natural" or "secular" frequencies:

$$
\omega_{n}=\left(2 n+\beta_{u}\right) \frac{\Omega}{2}, \quad n=0,1,2, \ldots
$$

The amplitudes of higher-frequency components are small compared with those at the fundamental $(n=0)$ frequency, $\omega_{0}=\beta_{u}(\Omega / 2)$. The high-frequency oscillating terms in the ion trajectory may therefore be neglected if $q_{u}$ is small, and the resulting ion trajectory may be written simply as:

$$
\begin{gathered}
u(t) \approx A \cos \omega_{u, 0} t+B \sin \omega_{u, 0} t \\
\omega_{u, 0}=\beta_{u} \frac{\Omega}{2}=\left(a_{u}+\frac{q_{u}^{2}}{2}\right)^{1 / 2} \frac{\Omega}{2} \\
u=x, y, \text { or } z
\end{gathered}
$$

Eq 1 may now be written as:

$$
\frac{d^{2} u}{d t^{2}}+\omega_{u, 0}^{2} u=0
$$

The ion equation of motion, eq 7, and its solution, eq 6 , describe simple harmonic oscillation along the $u$-axis. A harmonic oscillator may be considered a particle subject to a potential that varies quadratically with $u$. For a single ion trapped in a QIT, the potential experienced by the ion is generated by the sum of the $D C$ and RF voltages applied to the electrodes. The DC voltage produces an electrostatic potential and the average effect of the RF voltage may be regarded as arising from an additional static "potential." The combination of the potential produced by the RF voltage and the electrostatic potential from the $D C$ voltage defines the so-called pseudopotential, $\Phi_{0}$, [4]. Thus, the force, $\mathbf{F}=F_{x} \mathbf{i}+F_{y} \mathbf{j}+F_{z} \mathbf{k}$, acting on the ion is the product of ion charge and the negative gradient of the pseudopotential, or, in Cartesian components:

$$
\begin{aligned}
& F_{x}=m \frac{d^{2} x}{d t^{2}}=-q \frac{\partial \Phi_{0}}{\partial x} \\
& F_{y}=m \frac{d^{2} y}{d t^{2}}=-q \frac{\partial \Phi_{0}}{\partial y} \\
& F_{z}=m \frac{d^{2} z}{d t^{2}}=-q \frac{\partial \Phi_{0}}{\partial z}
\end{aligned}
$$

By substituting eq 7 into the above equation and rewriting it in terms of $\partial \Phi_{0} / \partial u$, we have:

$$
\begin{aligned}
& \frac{\partial \Phi_{0}}{\partial x}=-\frac{m}{q} \frac{d^{2} x}{d t^{2}}=\frac{m}{q} \omega_{x, 0}^{2} x \\
& \frac{\partial \Phi_{0}}{\partial y}=-\frac{m}{q} \frac{d^{2} y}{d t^{2}}=\frac{m}{q} \omega_{y, 0}^{2} y \\
& \frac{\partial \Phi_{0}}{\partial z}=-\frac{m}{q} \frac{d^{2} z}{d t^{2}}=\frac{m}{q} \omega_{z, 0}^{2} z
\end{aligned}
$$

By use of eq $1, \omega_{x, 0}^{2}, \omega_{y, 0}^{2}$, and $\omega_{x, 0}^{2}$ can be written as functions of $a_{u}$ and $q_{u}$ values:

$$
\begin{gathered}
\omega_{x, 0}^{2}=\omega_{y, 0}^{2}=\left(a_{x}+\frac{a_{x}^{2}}{2}\right)\left(\frac{\Omega}{2}\right)^{2} \\
\omega_{z, 0}^{2}=\left(-2 a_{x}+2 q_{x}^{2}\right)\left(\frac{\Omega}{2}\right)^{2}
\end{gathered}
$$

We may now integrate eqs 9 to obtain the pseudopotential, $\Phi_{0}$ :

$$
\begin{aligned}
\Phi_{0}(x, y, z)= & \frac{m}{2 q}\left(\frac{\Omega}{2}\right)^{2} \\
& \times\left(\left(a_{x}+\frac{q_{x}^{2}}{2}\right)\left(x^{2}+y^{2}\right)+\left(-2 a_{x}+2 q_{x}^{2}\right) z^{2}\right) \\
& + \text { constant } \\
= & D_{\rho} \frac{\rho^{2}}{\rho_{0}^{2}}+D_{z} \frac{z^{2}}{z_{0}^{2}}+\text { constant }
\end{aligned}
$$

in which

$$
\begin{gathered}
\rho^{2}=x^{2}+y^{2} \\
D_{p}=\frac{m \rho_{0}^{2}}{2 q}\left(\frac{\Omega}{2}\right)^{2}\left(a_{x}+\frac{q_{x}^{2}}{2}\right)
\end{gathered}
$$

and

$$
D_{z}=\frac{m z_{0}^{2}}{2 q}\left(\frac{\Omega}{2}\right)^{2}\left(-2 a_{x}+2 q_{x}^{2}\right)
$$

$D_{\rho}$ and $D_{z}$ represent the pseudopotential well depth in the radial and axial directions.

\section{Equilibrium Spatial Distribution of Ions}

At the $\sim 10^{-3}$ torr He pressure at which a QIT is typically operated, a given ion undergoes more than 10,000 collisions with neutrals per second. It is therefore reasonable that the ion distribution reaches equilibrium after a few seconds, because eq 11 shows that the pseudopotential exhibits a minimum at the center of the trap.

The pseudopotential approach is at best an approxi- 
mation, because the ions are driven continuously at the RF frequency. We can expect the approximation to be valid only when the $q_{u}$ value is reasonably small (e.g., 50.4 ). In addition, because we consider only the final equilibrium distribution, our result is independent of collision mechanisms and other relaxation processes. In other words, the distribution will depend only on the temperature of the system (ions or neutrals) not on the nature of the collision gas. However, the pseudopotential (unlike a mechanical harmonic oscillator) does depend on ion mass-to-charge ratio, and the resulting ion spatial distribution is also a function of ion mass-to-charge ratio.

For simplicity, we consider that all of the trapped ions have the same mass-to-charge ratio. At equilibrium, the ion number density (or ion spatial distribution) may then be written:

$$
n(x, y, z)=N \frac{\exp (-q \Phi(x, y, z) / k T)}{\int \exp (-q \Phi(x, y, z) / k T) d x d y d z}
$$

in which $N$ is the total number of trapped ions, $n$ is ion number density, $k$ is the Boltzmann constant, and $T$ is absolute temperature. In the denominator of eq 11, the integration covers all of three-dimensional space. $\Phi$ is the total potential acting on the ions and is the sum of the pseudopotential (eq 11) and the potential, $\Phi_{S}$. generated by the space charge.

$$
\Phi=\Phi_{0}+\Phi_{S}
$$

The space charge potential, $\Phi_{s}$ must satisfy Poisson's equation:

$$
\nabla^{2} \Phi_{S}=-\frac{q n}{\varepsilon_{0}}
$$

in which $\varepsilon_{0}$ is the dielectric constant in vacuo. Because the ion number density, $n$, depends in turn on the space charge potential, the above equation must be solved consistently with respect to eq 12 .

\section{Charge Spatial Distribution: Low Ion Density Limit}

If the number of ions is sufficiently small (i.e., $N \rightarrow 0$ ), the space charge potential, $\Phi_{s}$, is small compared with the pseudopotential. The contribution of the space charge potential may then be neglected in eq 11 and we obtain the space charge density:

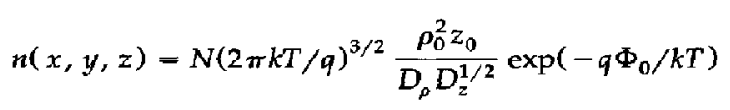

The space charge density is, therefore, a Gaussian distribution in all three dimensions (see eq 11a for $\Phi_{0}$ ). Interestingly, a recent experiment based on spatially resolved laser photodissociation demonstrated that the ion axial distribution (i.e., along the $z$-direction) indeed has a Gaussian profile [16].

\section{Charge Spatial Distribution: Maximum Ion Density Limit}

As the number of ions increases, the depth of the total potential well decreases (see below). The maximum number, $N=N_{\max }$, of ions is reached when the space charge potential increases to such an extent that the total potential well flattens to a constant value, $\boldsymbol{\Phi}_{T}$ :

$$
\Phi=\Phi_{0}+\Phi_{S}=\Phi_{T}
$$

In that limit, according to eq 11, the space charge number distribution also becomes constant:

$$
n_{0}=-\frac{\varepsilon_{0} \nabla^{2} \Phi_{S}}{q}=\frac{\varepsilon_{0} \nabla^{2} \Phi_{0}}{q}=\frac{\varepsilon_{0}}{q}\left(D_{p} \frac{4}{\rho_{0}^{2}}+D_{z} \frac{2}{z_{0}^{2}}\right)
$$

\section{Charge Spatial Distribution Under Intermediate Working Conditions}

In the usual intermediate range in which space charge contributes significantly to the total potential but does not flatten it completely, we need to solve eqs 12 to 14 consistently. First, we specify a spatially uniform ion distribution with a given ion number density and total number of charges. The potential of that charge distribution is then computed from eq 12, and a new ion charge distribution is computed from that potential by use of eq 14. The process is repeated until the potentials or distributions from two successive iterations agree with each other.

In general, the axial pseudopotential well depth, $D_{z}$, is not equal to the azimuthal radial well depth, $D_{\rho}$. The cylindrically symmetric charge distribution is therefore a two-dimensional function of $z$ and $\rho$, thereby increasing the computational complexity of the problem.

We used an operating condition of the QIT such that its pseudopotential becomes isotropic in all three spatial dimensions. The space charge potential and charge distribution thus depend only on distance from the center of the trap, and we have devised a simple computer program to solve the resulting onedimensional problem.

If the QIT pseudopotential is to exhibit spherical symmetry, then (see eq 11a):

$$
\frac{D_{p}}{\rho_{0}^{2}}=\frac{D_{z}}{z_{0}^{2}}
$$


or

$$
2\left(a_{x}+\frac{q_{x}^{2}}{2}\right)=\left(-2 a_{x}+2 q_{x}^{2}\right)
$$

in the usual case that $\rho_{0}^{2}=2 z_{0}^{2}$. The above condition then becomes:

$$
4 a_{x}=q_{x}^{2}
$$

The pseudopotential may now be written as:

$$
\begin{aligned}
\Phi_{0}(x, y, z) & =\frac{D_{\rho}}{\rho_{0}^{2}}\left(\rho^{2}+z^{2}\right)+\text { constant } \\
& =\frac{D_{\rho}}{\rho_{0}^{2}} r^{2}+\text { constant }
\end{aligned}
$$

in which $r=\rho^{2}+z^{2}$ is the distance from the center of the trap. Figure 1 shows the location of the curve, $4 a_{x}=q_{x}^{2}$, on the stability diagram. Because the pseudopotential is spherically symmetrical, the space charge distribution and its potential must also vary only with $r$. Determination of the three-dimensional space charge distribution and its associated space charge potential now reduces to a one-dimensional problem. We may therefore proceed to express the space charge distribution and Poisson's equation for the space charge potential (in spherical coordinates) as:

$$
n(r)=N \frac{\exp \left(-q\left(\Phi_{0}(r)+\Phi_{S}(r)\right) / k T\right)}{4 \pi \int \exp \left(-q\left(\Phi_{0}(r)+\Phi_{S}(r)\right) / k T\right) r^{2} d r}
$$

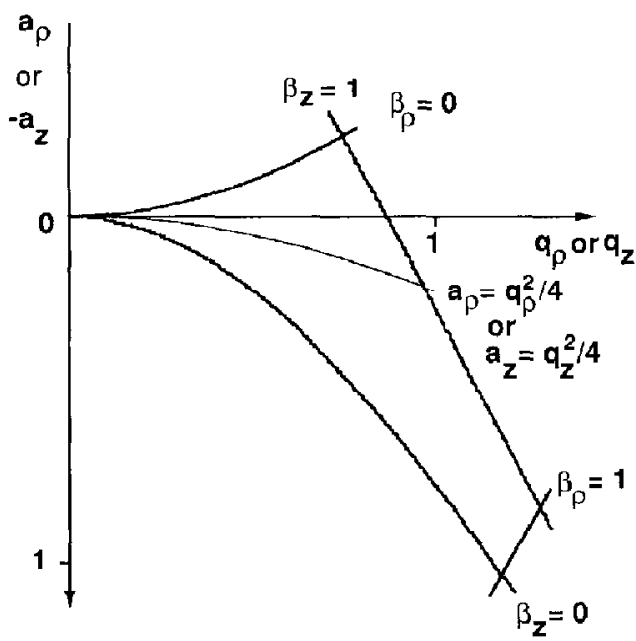

Figure 1. Stability diagram for a QIT. $a_{u}$ and $q_{u}(u=p$ or $z)$ are related to the $D C$ and RF voltages applied between the ring and endcap electrodes (see cq 1). Along the curve defined by $4 a_{2}=q_{z}^{2}$ or $4 a_{p}=a_{\rho}^{2}$, the pseudopotential and ion distribution have spherical symmetry, so that the ion spatial distribution depends only on distance from the center of the trap (see text). and

$$
\nabla^{2} \Phi_{s}=\frac{1}{r^{2}} \frac{d}{d r}\left(r^{2} \frac{d \Phi_{s}(r)}{d r}\right)=-q n(r) / \varepsilon_{0}
$$

As noted in the Theory section, eqs 20 and 21 cannot be solved by standard techniques for ordinary differential equations. We therefore developed the following iterative method. First, an initial ion distribution is specified (e.g., a spatially uniform distribution near the center of the trap), and its associated space charge potential is calculated. A new charge distribution is then generated by substituting the pseudopotential and the just-calculated space charge potential into eq 20. The iterations continue until the difference in the partition functions:

$$
P=4 \pi \int \exp \left(-q\left(\Phi_{0}(r)+\Phi_{S}(r)\right) / k T\right) r^{2} d r
$$

from two consecutive iterations is less than a specified value.

Specifically, eq 21 is solved by standard fourth-order Runge-Kutta numerical integration. A Fortran program was executed on a PC-compatible computer and data were transferred to a Macintosh $\Pi$ (Apple Computer, Inc., Cupertino, CA) for graphic processing. Typically, ten to 20 iterations were required to obtain less than 100 ppm change in the partition function for two consecutive iterations. We may now proceed to characterize the final charge spatial distribution and space charge potential.

\section{Results and Discussion}

\section{Effect of Total Number of Ions on Space Charge Distribution and Space Charge Potential}

Figure 2 shows a representative ion space charge distribution and how it depends on the total number of charges. We specified typical QIT operating conditions: $300 \mathrm{~K}$ and a pseudopotential of $1 \mathrm{~V}$, corresponding (for ions of $\mathrm{m} / \mathrm{z} 100$ ) to application of $1.334 \mathrm{VDC}$ and 163 $V_{(F-F)} R F$ voltage at $1.1 \mathrm{MHz}$ to the ring electrode (minimum ring radius, $\rho_{0}=1 \mathrm{~cm}$ ) with endcap electrodes $\left(z_{0}=1 / \sqrt{2} \mathrm{~cm}\right)$ grounded. Under these conditions, $q_{x}=0.0655 \leqslant 0.4$, so that the pseudopotential approximation should apply.

Figure 2 shows that as the number of ions increases, the charge density at the center of the trap (i.e., at $\rho=0$ ) increases, but not in direct proportion to the number of ions, and the width (e.g., at half-maximum height) of the charge density distribution also increases. It is important to recognize that because the pseudopotential magnitude varies with mass-to-charge ratio, the space charge distribution of ions of higher mass-to-charge ratio will be wider (not shown) than the distribution of an equal number of ions of lower mass-to-charge ratio. 


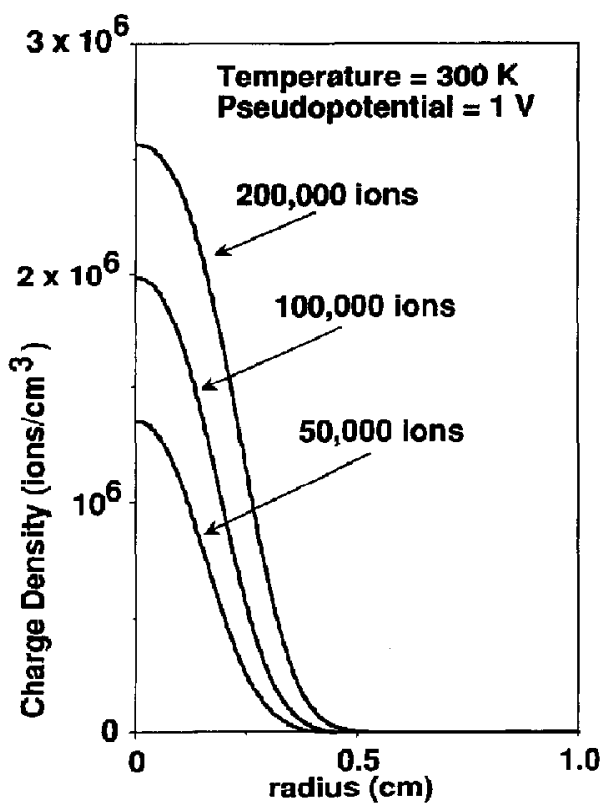

Figure 2. Dependence of ion radial distribution on number of ions.

Figure 3 shows that the ion space charge distribution varies with total number of ions because its corresponding spare charge potential also varies with ion number. Specifically, the pseudopotential produced by application of DC and RF voltages between ring and endcap electrodes in the absence of any trapped ions varies quadratically with distance away from the center of the trap. However, addition of ions to the trap adds a space charge potential whose effect is to flatten the total potential to a shallower well; the force (proportional to the slope of the potential curve) pushing ions toward the center of the trap is thus reduced, and ions spread out from the trap center. A second consequence of the distortion of the total potential is that the total potential no longer varies quadratically with distance from the center of the trap. As a result, the axial secular frequency now depends on ion position and is no longer a constant of the motion. Finally, since the ion spatial distribution is independent of any spatially constant potential, we chose $1 \mathrm{~V}$ as a common reference point for the total potential at $1-\mathrm{cm}$ radius (intersection of all four curves in Figure 3).

\section{Effect of Temperature on Space Charge Distribution and Space Charge Potential}

On decrease in temperature, ions tend to concentrate at the center of the trap, so that the charge density at the center of the trap increases and the width of the charge distribution decreases, as shown in Figure 4. The mag-

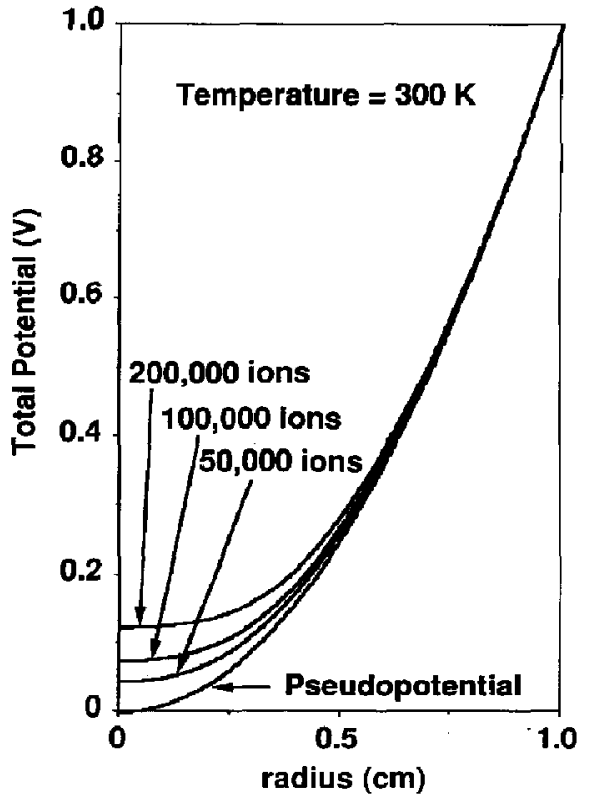

Figure 3. Dependence of total electrostatic potential (trap pseudopotential plus ion space charge potential) on number of ions.

nitude of these effects is comparable to that produced (Figure 2) by changing the number of ions by the same factor. Interestingly, changing the temperature does not dramatically change the total potential, as shown in Figure 5.

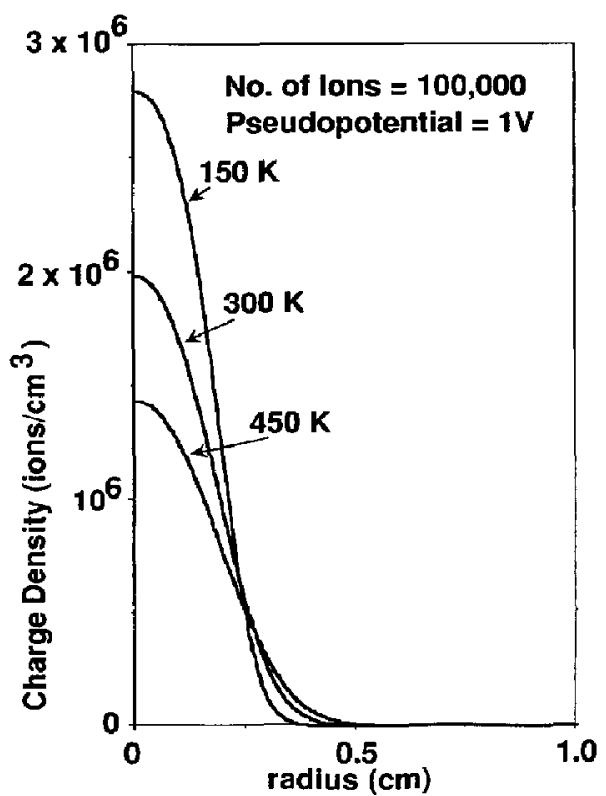

Figure 4. Dependence of ion radial distribution on temperature. 


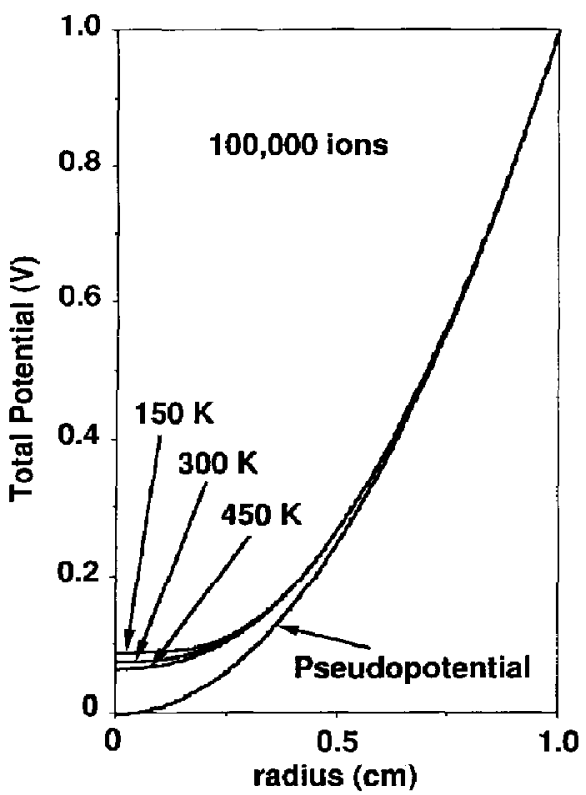

Figure 5. Dependence of total electrostatic potential (trap pseudopolenlial plus ion space charge potential) on temperature.

\section{Dependence on Pseudopotential}

The most dramatic change in the ion distribution is produced by changing the magnitude of the pseudopotential. Figure 6 shows that for 100,000 ions at $300 \mathrm{~K}$, the ion density at the center of the trap increases from $8 \times 10^{5}$ charges $/ \mathrm{cm}^{3}$ at a pseudopotential of $0.5 \mathrm{~V}$ to $>4.5 \times 10^{6}$ charges $/ \mathrm{cm}^{3}$ at a pseudopotential of $2 \mathrm{~V}$.

\section{Conclusions}

The present calculations offer a semiquantitative basis for analyzing the effect of ion number, temperature, and pseudopotential on the distribution of ions (and their associated space charge potential) in a QIT. The distribution of trapped and thermalized ions of a single mass-to-charge ratio is also described. Future work will extend the computation to the distribution for ions of differenl mass-lo-chaige ralios. These calculations provide a basis for comparing theory to experiment (e.g., recent determinations of the average kinetic energy of ions in a QIT $[17,18]$ ) and for future allempls to establish a QIT mass calibration law that incorporates the effect of space charge.

Extension of the present method to the development of a new and accurate mass calibration law is a difficult task, which requires understanding of time evolution of the distribution and effects of excitation and ejection on the ion distribution. For example, ejection by mass-selective instability [19] at $q_{z}=0.908$ (compared to $q_{z} \leq 0.4$ here) excites ions to energies of

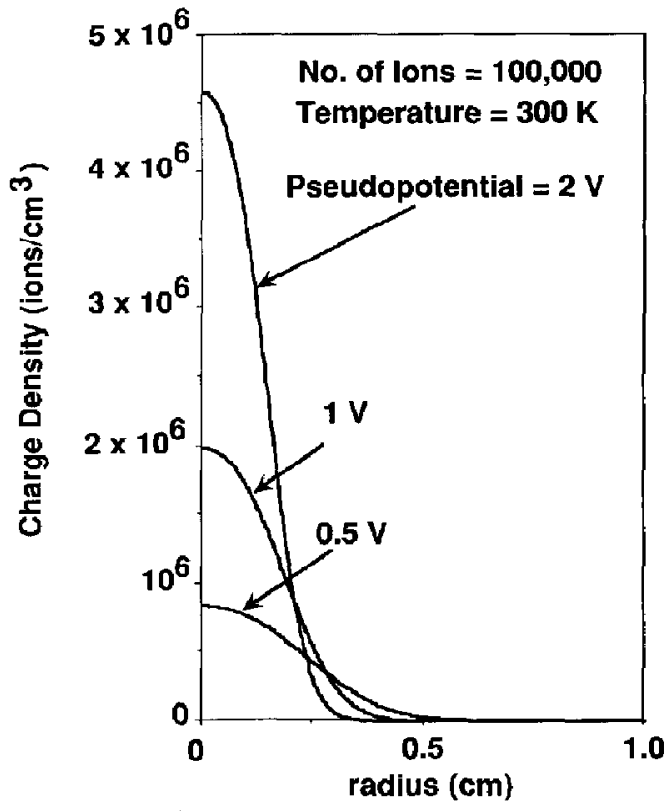

Figure 6. Dependence of ion radial distribution on trap pseudopotential (i.e, the effective electrostatic potential generated by application of DC and RF voltages between the ring and end cap electrodes).

several kiloelectron volts. However, resonant axial ejection [20] at ion energies of tens to hundreds of electron volfs can be conducted at lower $q_{z}$ value, and the present method provides a description of the spatial distribution of the remaining unejected ions in that case. In any case, we have provided a first step in predicting the effect of space charge on the dimensions of the ion cloud and on the shape of the trapping well -a starting point for the long journey to precise mass analysis in a QIT.

\section{Acknowledgments}

This work was supported by grants (to A.C.M.) from the National Science Foundation (CHE-9021058), The Ohio State University, and the National High Magnetic Field Laboratory at Florida State University.

\section{References}

1. March, R. E.; Hughes, R. J. Quadrupole Storage Mass Spectrometry; Wiley: New York, 1989.

2. Cooks, R. G.; Kaiser Jr., R. E. Acc. Chem. Res. 1990, 23, 213-219.

3. Tudd, J. F. J. Muss Spectrom. Rev. 1991, 10, 3-52.

4. Dehmelt, H. G. Adv. At. Mol. Phys. 1967, 3, 53-72.

5. Stafford Jr., G. C.; Kelly, P. E.; Syka, J. E. P.; Reynolds, W. E.; Todd, J. F. J. Int. I. Mass Spectrom. Ion Phys. 1984, 60, 85-98.

6. Vedel, F.; Andre, J.; Vedel, M,; Brincourt, G. Phys. Rev. A 1983, 27, 2321-2330.

7. Todd, J. F. J-; Waldren, R. M,; Mather, R. E. Int. I. Mass Spectrom. Ion Phys. 1980, 34, 325-349. 
8. Fischer, E. Z. Phys. 1959, 156, 26.

9. Schwebel, C.; Möller, P. A.; Manh, P. T. Rev. Phys. Appl. 1975, 10, 227

10. Todd, J. F. J.; Waldren, R. M.; Freer, D. A.; Turner, R. B. Int. J. Mass Spectrom. Ion Phys. 1980, 35, 107-150.

11. Vedel, F.; Andre, J. Phys. Rev. A 1984, 29, 2098-2101.

12. Vedel, F. Int. J. Mass Spectrom. Ion Processes 1991, 106, 33-61.

13. Helmer, J. C. In Proceedings of the 39th Annual ASMS Conference on Mass Spectrometry and Allied Topics; Nashville, TN, 1991; pp 528-529.

14. Knight, R. D. Int. J. Mass Spectrom. Ion Processes 1983, 51, $127-131$.
15. McLachlan, N. W. Theory and Applicutions of Mathieu Functions; Clarendon: Oxford, 1947.

16. Hemberger, P. H.; Nogar, N. S.; Williams, J. D.; Cooks, R. G.; Syka, J. E. P. Chem. Phys. Lett. 1992, 191, 405-410.

17. Nourse, B. D.; Kenttäma a, H. I. J. Phys. Chem. 1990, 94 5809-5812.

18. Basic, C.; Eyler, J. R.; Yost, R. A. I. Am. Soc. Mass Spectrom. 1992, 3, 716-726.

19. Kelley, P. E.; Stafford Jr., G. C.; Stephens, D. R. U.S. Patent 4,540,884, September 10, 1985.

20. Armitage, M. A.; Fulford, J. E.; Duong, N.-H.; Hughes, R. I.; March, R. E. Can. J. Chem. 1979, 57, 2108-2113. 